\title{
THE HYPERSPACES OF SUBCONTINUA OF THE PSEUDO-ARC AND OF SOLENOIDS OF PSEUDO-ARCS ARE CANTOR MANIFOLDS
}

\author{
PAWEL KRUPSKI
}

(Communicated by James E. West)

\begin{abstract}
New proofs of the above facts are based on specific homogeneity properties of the pseudo-arc and of solenoids of pseudo-arcs.
\end{abstract}

The reader is referred to [5] for hyperspace theory. It is known that if $X$ is the pseudo-arc or a solenoid of pseudo-arcs (see [7] for the definition), then the hyperspace $C(X)$ of all nonvoid subcontinua of $X$ is 2-dimensional. It is proved in [6] that if $X$ is the pseudo-arc, then $C(X)$ is also a Cantor manifold, i.e., no 0-dimensional subset separates $C(X)$. In [2] a general theorem is presented that $C(X)$ has this property for an arbitrary metric, nondegenerate continuum $X$. Our proof of the theorem in the title is an application of the following result [3].

Lemma 1. If $X$ is an n-dimensional, locally compact, connected, homogeneous, metric space, then no $(n-2)$-dimensional subset separates $X(n \geq 1)$.

Lemma 2. If a dense, connected subset of a metric separable space $X$ is separated by no $n$-dimensional subset, then the space $X$ has the same property.

(1) Let $X$ be the pseudo-arc. To show that $C(X)$ is a Cantor manifold it suffices to observe, by Lemmas 1 and 2, that the subspace $Y \subset C(X)$ of all nondegenerate, proper subcontinua of $X$ is connected, locally compact, homogeneous (see [1]), as well as 2-dimensional and dense.

(2) Let $X$ be a solenoid of pseudo-arcs with the continuous decomposition $D$ into pseudo-arcs such that $X / D$ is a solenoid $S$. The set $D$ as a subspace of $C(X)$ is homeomorphic to $S$. As in (1) the open subspace $Y$ of $C(X)$ is connected and dense. The set $Y \backslash D$ is dense in $Y$ and is the union of two disjoint, open, connected, 2-dimensional subsets $M=\{y \in Y: d \neq y \subset d \in D\}$

Received by the editors February 25, 1989 and, in revised form, November 19, 1989.

1980 Mathematics Subject Classification (1985 Revision). Primary 54B20; Secondary 54F45.

Key words and phrases. Pseudo-arc, solenoid of pseudo-arcs, hyperspace of subcontinua, homogeneous space, Cantor manifold.

This note was written while the author was visiting the Department of Mathematics, University of Helsinki. 
and $N=\{y \in Y: d \neq y \supset d \in D\}$. It follows from [4] and from properties of solenoids of pseudo-arcs [7] that for every pair $y_{1}, y_{2} \in M\left(y_{1}, y_{2} \in N\right)$ there exists a homeomorphism $h: X \rightarrow X$ such that $h\left(y_{1}\right)=h\left(y_{2}\right)$. The induced homeomorphism $\hat{h}: C(X) \rightarrow C(X)$ satisfies $\hat{h}(M)=M, \hat{h}(N)=N$ and $\hat{h}\left(y_{1}\right)=y_{2}$, so both $M$ and $N$ are homogeneous and, by Lemma 1, no 0-dimensional subset separates neither $M$ nor $N$. Suppose a 0-dimensional subset $C$ separates $Y$. Without loss of generality we may assume that $C$ is a closed subset of $Y$. It means $Y \backslash C=A \cup B$, where $A, B$ are nonvoid, disjoint and open subsets of $C(X)$. In view of the above properties of $M$ and $N$ we may assume $M \subset A$ and $N \subset B$. Thus $C \subset D$. If there is $d \in D \backslash C$, then some order arc $\alpha \subset C(X)$ passing through $d$ joins $M$ and $N$, which is impossible, since $\alpha \cap D=\{d\}$ and $C$ separates $Y$ between $M$ and $N$. Therefore $C=D$, hence $C$ is not 0-dimensional, a contradiction.

Remark. A similar proof works for $X$ being a solenoid. However in this case $C(X)$ is the cone over $X$, which is evidently a Cantor manifold.

\section{REFERENCES}

1. H. Cook, A locally compact, homogeneous metric space which is not bihomogeneous, Topology Proc. 11 (1986), 25-27.

2. J. Krasinkiewicz, No 0-dimensional set disconnects the hyperspace of a continuum, Bull. Acad. Polon. Sci. 19 (1971), 755-758.

3. P. Krupski, Homogeneity and Cantor manifolds, Proc. Amer. Math. Soc. (to appear).

4. G. R. Lehner, Extending homeomorphisms on the pseudo-arc, Trans. Amer. Math. Soc. 98 (1961), 369-394.

5. S. B. Nadler, Jr., Hyperspaces of sets, Marcel Dekker, New York, 1978.

6. T. Nishiura and C. J. Rhee, The hyperspace of a pseudo-arc is a Cantor manifold, Proc. Amer. Math. Soc. 31 (1972), 550-556.

7. J. T. Rogers, Jr., Solenoids of pseudo-arcs, Houston J. Math. 3 (1977), 531-537.

Mathematical Institute, University of Wroclaw, Pl. Grunwaldzki 2/4, 50-384 Wroclaw, Poland 